\title{
Optimal Diagnostic Indices for Idiopathic Normal Pressure Hydrocephalus Based on the 3D Quantitative Volumetric Analysis for the Cerebral Ventricle and Subarachnoid Space
}

\author{
(D) S. Yamada, M. Ishikawa, and (D) K. Yamamoto
}

\begin{abstract}
BACKGROUND AND PURPOSE: Despite the remarkable progress of 3D graphics technology, the Evans index has been the most popular index for ventricular enlargement. We investigated a novel reliable index for the MR imaging features specified in idiopathic normal pressure hydrocephalus, rather than the Evans index.
\end{abstract}

MATERIALS AND METHODS: The patients with suspected idiopathic normal pressure hydrocephalus on the basis of the ventriculomegaly and a triad of symptoms underwent the CSF tap test. CSF volumes were extracted from a T2-weighted 3D spin-echo sequence named "sampling perfection with application-optimized contrasts by using different flip angle evolutions (SPACE)" on 3T MR imaging and were quantified semiautomatically. Subarachnoid spaces were divided as follows: upper and lower parts and 4 compartments of frontal convexity, parietal convexity, Sylvian fissure and basal cistern, and posterior fossa. The maximum length of 3 axial directions in the bilateral ventricles and their frontal horns was measured. The "z-Evans Index" was defined as the maximum z-axial length of the frontal horns to the maximum cranial z-axial length. These parameters were evaluated for the predictive accuracy for the tap-positive groups compared with the tap-negative groups and age-adjusted odds ratios at the optimal thresholds.

RESULTS: In this study, 24 patients with tap-positive idiopathic normal pressure hydrocephalus, 25 patients without response to the tap test, and 23 age-matched controls were included. The frontal horns of the bilateral ventricles were expanded, with the most excessive expansion being toward the z-direction. The CSF volume of the parietal convexity had the highest area under the receiver operating characteristic curve (0.768), the z-Evans Index was the second (0.758), and the upper-to-lower subarachnoid space ratio index was the third (0.723), to discriminate the tap-test response.

CONCLUSIONS: The CSF volume of the parietal convexity of $<38 \mathrm{~mL}$, upper-to-lower subarachnoid space ratio of $<0.33$, and the z-Evans Index of $>0.42$ were newly proposed useful indices for the idiopathic normal pressure hydrocephalus diagnosis, an alternative to the Evans Index.

ABBREVIATIONS: AUC $=$ area under the receiver operating characteristic curve; DESH $=$ disproportionately enlarged subarachnoid space; iNPH $=$ idiopathic normal pressure hydrocephalus; SPACE = sampling perfection with application-optimized contrasts by using different flip angle evolutions

diopathic normal pressure hydrocephalus (iNPH) has been diagnosed since the evidence-based guidelines for diagnosis and management of iNPH were announced in Japan, the United States, and Europe. ${ }^{1-5}$ Frequently, patients with iNPH have shortstepped gaits at first, followed by cognitive impairment and urinary incontinence. The Study of iNPH on Neurologic Improve-

Received March 4, 2015; accepted after revision May 1.

From the Normal Pressure Hydrocephalus Center (S.Y., M.I.) and Department of Neurosurgery and Stroke Center (S.Y., M.I., K.Y.), Rakuwakai Otowa Hospital, Kyoto, Japan.

Please address correspondence to Shigeki Yamada, MD, PhD, Normal Pressure Hydrocephalus Center, Department of Neurosurgery and Stroke Center, Rakuwakai Otowa Hospital, Otowachinji-cho 2, Yamashina-ku, Kyoto 607-8602, Japan; e-mail: shigekiyamada3@gmail.com

-- Indicates open access to non-subscribers at www.ajnr.org

$\equiv$ Indicates article with supplemental on-line table.

http://dx.doi.org/10.3174/ajnr.A4440 ment (SINPHONI) showed that narrow sulci at the high convexity and an enlarged Sylvian fissure with ventricular dilation, which was designated as "disproportionately enlarged subarachnoid-space hydrocephalus (DESH)," were important MR imaging features for iNPH diagnosis. ${ }^{6}$ The SINPHONI also confirmed that a lumbar CSF tap test was a necessary diagnostic test for probable iNPH and predicted a favorable response to a ventriculoperitoneal shunt surgery. ${ }^{7}$

Despite the remarkable progress of 3D graphics technology, the Evans Index proposed by William Evans in 1942 has been the most popular index of ventricular enlargement, ${ }^{8}$ and an Evans Index of $>0.3$ has been adopted as a criterion for ventriculomegaly in the Japanese and international iNPH guidelines. ${ }^{1-5}$ However, some studies using volumetric analysis suggested that it was not a sufficient linear index for evaluating ventricular enlargement. ${ }^{9,10}$ In recent years, a T2-weighted 3D spin-echo se- 
quence with sampling perfection with application-optimized contrasts by using different flip angle evolutions (SPACE sequence; Siemens, Erlangen, Germany) has been developed. ${ }^{11-14}$ This volumetric sequence enables the decrease of specific absorption rate limits and a scan of the whole brain in a single slab and a true isotropic $3 \mathrm{D}$ data record with high resolution (voxel size $\leq 1$ $\mathrm{mm}^{3}$ without interpolation). Taking advantage of the high sensitivity to detect CSF on the T2-weighted 3D-SPACE sequence, a new automated segmentation technique by using a simple threshold algorithm has been developed. ${ }^{15}$ The aim of the present study was to investigate the association between several $1 \mathrm{D}$ and $3 \mathrm{D}$ parameters of the ventricles and subarachnoid space and the response to the CSF tap test in patients with suspected iNPH in a systematic manner.

\section{MATERIALS AND METHODS Study Population}

The study design and protocol were approved by the ethics committee for human research at our hospital. We prospectively collected the patients for $3 \mathrm{~T}$ MR imaging beginning in November 2013, when the best protocols of imaging acquisition and extraction of ventricular and subarachnoid CSF were determined. $\mathrm{Pa}-$ tients 60 years of age or older who had ventriculomegaly and symptoms of short-stepped gait and/or cognitive impairment were referred to our iNPH center as having suspected iNPH by neurologists and neurosurgeons around Kyoto. The comorbidities, including Alzheimer disease and cerebrovascular diseases, were diagnosed by the neurologists before referral to our iNPH center and were confirmed on MR imaging and ${ }^{123} \mathrm{I}-\mathrm{N}$-isopropylp-iodoamphetamine-SPECT in our center. Forty-nine consecutive patients with suspected iNPH underwent CSF removal of 30 $\mathrm{mL}$ via a lumbar tap (CSF tap test) concurrently with a T2weighted 3D-SPACE sequence on $3 \mathrm{~T}$ MR imaging and ${ }^{123} \mathrm{I}-\mathrm{N}$ isopropyl-p-iodoamphetamine-SPECT. According to the Japanese iNPH guidelines, ${ }^{4}$ improvements of symptoms were assessed by the iNPH grading scale and the quantitative examination of gait and cognition before and at 1 day and 4 days after the CSF tap test. Gait was assessed with a 3-m Timed Up and Go Test and a 10 -m straight walk (time in seconds and number of steps). Cognition was assessed by the Mini-Mental State Examination, the Frontal Assessment Battery, and the Trail-Making Test. Clinical improvement was defined as $\geq 10 \%$ improvement of the best changes in any of the quantitative examinations or $\geq 1$ point of improvement of the iNPH grading scale. On the basis of the response to the CSF tap test, 49 patients with suspected iNPH were divided into 24 patients with positive response to the tap test and 25 with negative response to the tap test. Additionally, 23 agematched controls consisting of the healthy volunteers or patients who were 60 years of age or older who did not have ventriculomegaly, the classic triad of iNPH, a massive intracranial lesion, or a fluid collection such as subdural hematoma underwent a T2weighted 3D-SPACE sequence with informed written consent. Patients diagnosed with secondary normal pressure hydrocephalus or congenital/developmental etiology normal pressure hydrocephalus were excluded from this study. Seventy-two patients (mean age, $76.8 \pm 6.8$ years; range, 61-89 years; 46 men, 26 women) were included in this study.

\section{Image Acquisition}

All MR imaging examinations were performed with a 64-channel 3T MR imaging system (Magnetom Skyra; Siemens). We preliminarily examined the most adequate $\mathrm{TR}$ and TE of the T2weighted 3D-SPACE sequence for CSF discrimination. The parameters of the T2-weighted 3D-SPACE sequence were set at TR, $2800 \mathrm{~ms}$; TE, $286 \mathrm{~ms}$; section thickness, $0.9 \mathrm{~mm}$ with 192 sections in a single slab; FOV, $230 \mathrm{~mm}$; bandwidth, $789 \mathrm{~Hz} / \mathrm{Px}$; matrix (pixel size), $192 \times 192$; voxel size, $0.6 \times 0.6 \times 0.9 \mathrm{~mm}$. Image acquisition time was 4 minutes 16 seconds.

\section{Segmentation and Quantification of the Ventricular and Subarachnoid Space}

The sagittal source images of T2-weighted 3D-SPACE were automatically processed to create $3 \mathrm{D}$ volume-rendering reconstruction and MPR images by using an independent 3D volume-analyzer workstation (SYNAPSE 3D; Japanese local name, SYNAPSE VINCENT; Fujifilm Medical Systems, Tokyo, Japan). The intracranial volume was segmented by the use of the combined techniques of the edge-guided nonlinear interpolation and usersteered live-wire segmentation. ${ }^{16,17}$ After that, the CSF spaces were automatically segmented from brain parenchyma by using a simple threshold algorithm (Fig 1). ${ }^{15}$ The threshold range for the signal intensity of CSF on the T2-weighted 3D-SPACE sequence of 3T MR imaging was extremely stable at $650-700$ of the lower limit threshold and no upper limit threshold. The bilateral, third, and fourth ventricles were manually segmented, respectively, and they were subsequently combined as a total ventricle.

Subarachnoid spaces were automatically segmented as the total intracranial CSF space minus a total ventricle. Furthermore, subarachnoid spaces were divided into the upper and lower parts in a horizontal section on the anterior/posterior commissure plane at the level of the junction point of the vein of Galen and the straight sinus. The upper-to-lower subarachnoid space ratio was defined as the upper part to the lower part of the subarachnoid spaces (Fig 2). In addition, the subarachnoid space was divided into 4 parts, frontal convexity, parietal convexity, Sylvian fissure and basal cistern, and posterior fossa, by using the manual segmentation technique, as shown in Fig 3. The parietal convexity was defined as the posterior part from the central sulcus.

The labeling of the segmented volumes was measured by counting the number of voxels automatically. The volume ratios of the ventricles and subarachnoid spaces (\%) were calculated as ratios of the ventricle volumes to the intracranial volume. To evaluate the validity of the measured volumes, we segmented and measured the ventricles and subarachnoid spaces in the first 11 consecutive patients by using the SYNAPSE 3D workstation and the open-source 3D Slicer software package (www.slicer.org). ${ }^{18}$ The Pearson correlation coefficients among the 2 software packages were 0.838 for a total intracranial CSF space and 0.989 for the total ventricular volumes.

\section{D Coordinates of the Bilateral Ventricle}

Three axes for the spatial coordinates of the head position were used as follows: $\mathrm{x}$ is the left/right dimension, $\mathrm{y}$ is the posterior/ anterior dimension, and $\mathrm{z}$ is the ventral/dorsal or inferior/superior dimension. The $\mathrm{x}$ and $\mathrm{z}$ dimensions were perpendicular, and

AJNR Am J Neuroradiol 36:2262-69 Dec 2015 www.ajnr.org 

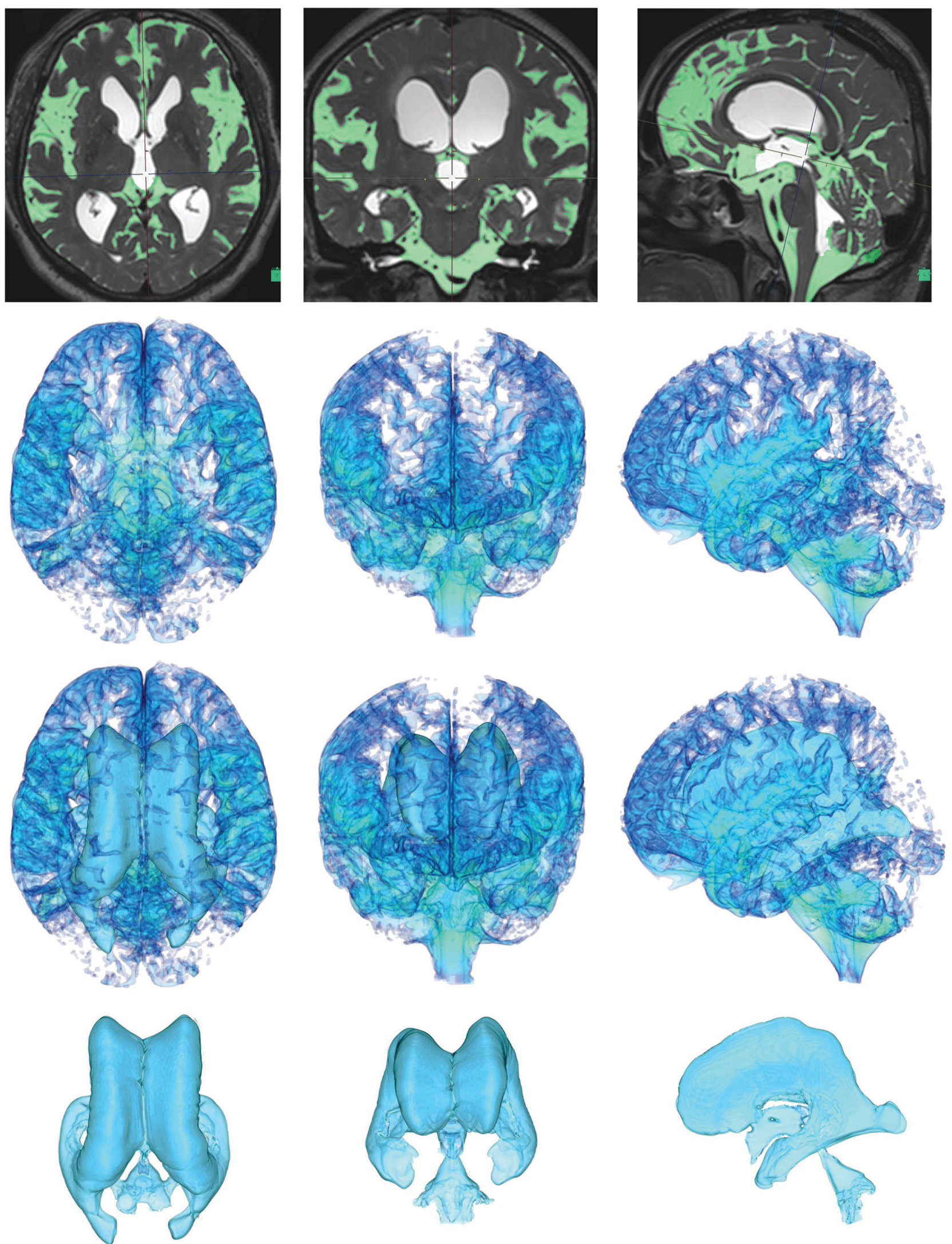

FIG 1. Automatic extraction of CSF space. The figures in the top row show the MIP images on the T2-weighted 3D-SPACE sequence in the representative iNPH case. Light green indicates the subarachnoid space segmented automatically at a threshold intensity of $>700$ on the SYNAPSE 3D workstation. The other figures show the 3D volume-rendering reconstruction images of the subarachnoid space on the second line, total CSF on the third line, and ventricles on the last line. The left, middle, and right column figures show axial, coronal, and sagittal dimensional views, respectively. 
the y dimension was parallel to the anterior/posterior commissure line. The Evans Index was measured as the maximal width of the frontal horns of the bilateral ventricles to the maximal width of the internal diameter of the cranium on the basis of the $x$ dimension. ${ }^{9,10}$ The $\mathrm{z}$-Evans Index was defined as the maximum $\mathrm{z}$-axial length of the frontal horn, which was between the roof and bottom of the larger lateral ventricle to the maximum cranial $\mathrm{z}$-axial length at the base of the posterior end of the foramen of Monro (Fig 4). In the same procedure, the $y$-Evans Index was defined as the maximum y-axial length between the posterior end of the foramen of Monro and the anterior end of the frontal horns to the maximum cranial y-axial length. Additionally, x-, $y$-, and z-Maximum Indices were measured as the maximum width of the bilateral ventricles to the maximum internal cranium width on each of 3 dimensions, as shown in Fig 4.

\section{Statistical Analysis}

The prevalence ratios of high-convexity tightness, enlarged Sylvian fissure, and comorbidities such as Alzheimer disease or dementia with Lewy bodies, Parkinsonism, cerebrovascular diseases, narrow spinal canal, and disuse muscle atrophy were compared among the 3 groups by the $\chi^{2}$ test. Mean values and SDs for age and 3D and 1D indices among the tap-positive, tap-negative,

FIG 2. Division of the subarachnoid space into the upper and lower parts. The subarachnoid space was divided into the upper and lower parts in a horizontal section on the anterior/posterior commissure plane at the level of the junction point of the vein of Galen and the straight sinus. The left and right figures show coronal and sagittal views. The Sylvian fissure is typically included in the lower part of the subarachnoid space. The upper-to-lower subarachnoid space ratio was defined as the upper part to the lower part of the subarachnoid space.
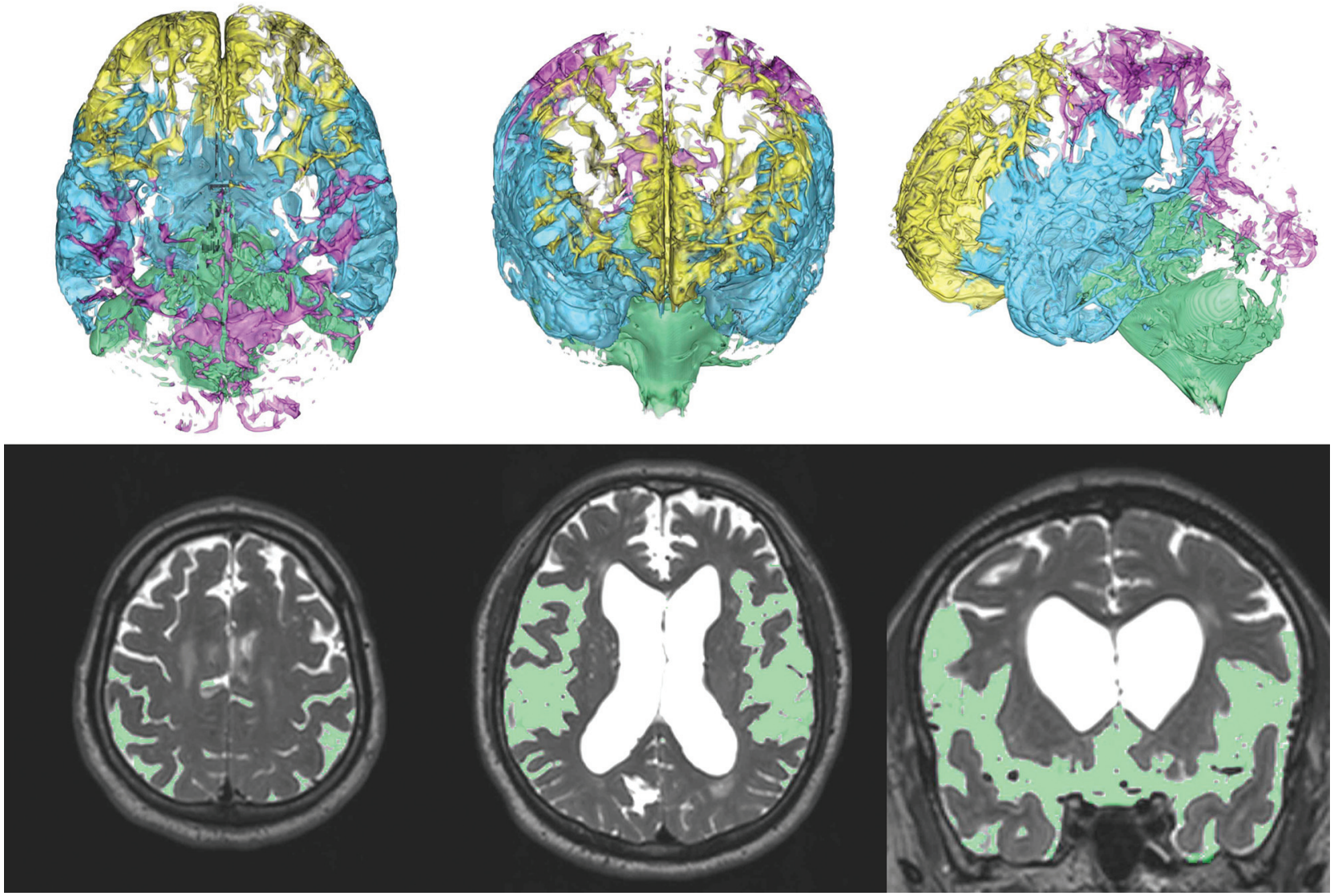

FIG 3. Division of the subarachnoid space into the 4 parts. The subarachnoid space was divided into the following 4 parts: frontal convexity (yellow), parietal convexity (magenta), Sylvian fissure and basal cistern (sky blue), and posterior fossa (light green) in the 3D segmentation. The left, middle, right upper 3D volume-rendering reconstruction images show the axial, coronal, and sagittal dimensional views, respectively. Light green in the left lower axial MIP image indicates the segmented region of the parietal convexity of the subarachnoid space, and that in the middle and right lower MIP images indicates axial and coronal views of the Sylvian fissure and basal cistern. 


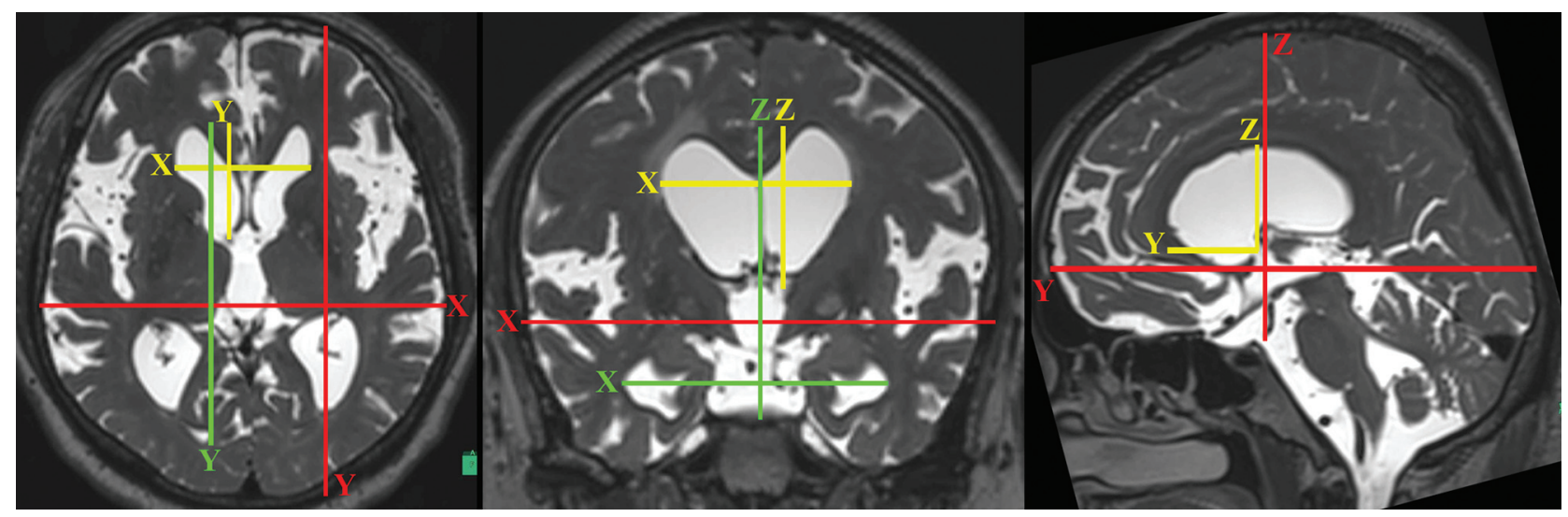

FIG 4. Three-directional linear indicators for evaluating the size of bilateral ventricles. The figures are the 3-directional MPR reconstruction images of the T2-weighted 3D SPACE. On the basis of the anterior/posterior commissure line, $x-$-, $y$-, and $z$-axes for spatial coordinates of head position were defined. The green lines show the maximum length of 3 -axial directions of the bilateral ventricles. The yellow lines show the maximum 3-axial length of the frontal horn of the bilateral ventricles. The red lines show the maximum intracranial 3-axial width. In addition to the original Evans Index, the $y$ - and z-Evans Indices were defined as the maximum length from the foramen of Monro to the anterior and superior extremities of the frontal horns (yellow lines)/the maximum intracranial $y$ - and z-axial length (red lines), respectively. The $x-, y-$, and $z-M a x i m u m$ Indices were defined as the maximum width of the bilateral ventricles (green lines)/the maximum intracranial width on the each of the 3 dimensions (red lines).

Table 1: Clinical characteristics of the study population

\begin{tabular}{|c|c|c|c|c|c|c|c|}
\hline & $\begin{array}{l}\text { No. }(\%) \\
\text { (72 Total) }\end{array}$ & $\begin{array}{c}\text { No. (\%) } \\
\text { (24 Tap-Positive) }\end{array}$ & $P$ Value $e^{\text {la }}$ & $P$ Value $^{2 b}$ & $\begin{array}{c}\text { No. (\%) } \\
\text { (25 Tap-Negative) }\end{array}$ & $P$ Value $^{3 c}$ & $\begin{array}{c}\text { No. }(\%) \\
\text { (23 Controls) }\end{array}$ \\
\hline Male sex & $46(64 \%)$ & $15(62 \%)$ & 1.000 & .917 & $17(68 \%)$ & .831 & $14(61 \%)$ \\
\hline Evans Index $>0.3$ & $42(58 \%)$ & $19(79 \%)$ & $.005^{\mathrm{d}}$ & .252 & $15(60 \%)$ & .145 & $8(35 \%)$ \\
\hline Callosal angle $<90^{\circ}$ & $46(64 \%)$ & $21(88 \%)$ & $<.001^{\mathrm{d}}$ & .962 & $23(92 \%)$ & $<.001^{\mathrm{d}}$ & $2(9 \%)$ \\
\hline High-convexity tightness & $35(49 \%)$ & $19(79 \%)$ & $<.001^{\mathrm{d}}$ & .154 & $14(56 \%)$ & $.002^{\mathrm{d}}$ & $2(9 \%)$ \\
\hline Enlarged Sylvian fissure & $33(46 \%)$ & $19(79 \%)$ & $<.001^{\mathrm{d}}$ & .154 & $14(56 \%)$ & $<.001^{d}$ & 0 \\
\hline Alzheimer disease & $22(31 \%)$ & $4(17 \%)$ & .666 & $.042^{d}$ & $12(48 \%)$ & .205 & $6(26 \%)$ \\
\hline Parkinsonism & $5(7 \%)$ & $2(8 \%)$ & .489 & 1.000 & $3(12 \%)$ & .263 & 0 \\
\hline Cerebrovascular diseases & $15(21 \%)$ & $1(4 \%)$ & $.022^{\mathrm{d}}$ & .115 & $6(24 \%)$ & .615 & $8(35 \%)$ \\
\hline Narrow spinal canal & $7(10 \%)$ & $3(13 \%)$ & .248 & 1.000 & $4(16 \%)$ & .139 & 0 \\
\hline Muscle atrophy of lower leg & $12(17 \%)$ & $5(21 \%)$ & .065 & .802 & $7(28 \%)$ & $.019^{d}$ & 0 \\
\hline Mean age ( $\pm S D)(y r)$ & $76.8( \pm 6.8)$ & $76.2( \pm 7.5)$ & .741 & .541 & $77.3( \pm 5.4)$ & .893 & $76.8( \pm 7.8)$ \\
\hline
\end{tabular}

a $P$ value: probability value for the $\chi^{2}$ test between the tap-positive group and controls.

${ }^{\mathrm{b}} P$ value ${ }^{2}$ : probability value for the $\chi^{2}$ test between the tap-positive and tap-negative groups.

${ }^{c} P$ value ${ }^{3}$ : probability value for the $\chi^{2}$ test between tap-negative group and controls.

d Significant.

and control groups were calculated and compared in each group by the Mann-Whitney-Wilcoxon test. The volumes and volume ratios of the total ventricles, bilateral ventricles and total subarachnoid space, the 4 segmented parts of the subarachnoid spaces, upper-to-lower subarachnoid space ratio, 3-directional linear 1D indices of the bilateral ventricles, and the callosal angle were calculated as the area under the receiver operating characteristic curves (AUC) to evaluate the optimal thresholds to maximize the sum of sensitivities and specificities for differentiation between the tap-positive and the tap-negative groups. Using the optimal thresholds from AUC analyses, we calculated age-adjusted ORs and 95\% CIs for comparing the tap-positive group with the tap-negative group in a multivariate logistic regression model. Additionally, 14 patients with shunt-effective iNPH were evaluated, and their 3D and 1D indices were compared to 25 patients without response to the tap test. The relationships between the 2 indices were compared by using the Pearson correlation coefficient $(r)$. Age was treated as a continuous variable for all statistical analyses. All missing data were treated as deficit data that did not affect other variables. Statistical significance was as- sumed at $P<.05$. Statistical analysis was performed by using $\mathrm{R}$ software (Version 3.0.1; http://www.R-project.org).

\section{RESULTS}

\section{Clinical Characteristics}

An Evans Index of $>0.3$, callosal angle of $<90^{\circ}$, narrow sulci at the high convexity, and an enlarged Sylvian fissure, which were conventional morphologic indices for iNPH diagnosis, were significantly different between the tap-positive group and the controls, but there was not any statistical significance between the tappositive and tap-negative groups (Table 1). The tap-negative group had a higher frequency of Alzheimer disease (48\%) and cerebrovascular diseases (26\%) compared with the tap-positive group.

\section{Parameters Associated with CSF Tap-Test Responses}

Table 2 shows the result of the mean values of the 3D and 1D indices. Among the 3D indices, volume ratios of the total ventricle or bilateral ventricles, CSF volume of the total subarachnoid space or parietal convexity, and upper-to-lower subarachnoid space ra- 
Table 2: Parameters among the tap-positive, tap-negative, and control groups

\begin{tabular}{|c|c|c|c|c|c|c|c|}
\hline & $\begin{array}{c}\text { Mean } \\
\text { (72 Total) }\end{array}$ & $\begin{array}{l}\text { Mean (24 Tap- } \\
\text { Positive) }\end{array}$ & $\begin{array}{c}P \\
\text { Value }^{\text {la }}\end{array}$ & $\begin{array}{c}P \\
\text { Value }^{2 b}\end{array}$ & $\begin{array}{c}\text { Mean (25 Tap- } \\
\text { Negative) }\end{array}$ & $\begin{array}{c}P \\
\text { Value }^{3 c}\end{array}$ & $\begin{array}{c}\text { Mean } \\
\text { (23 Controls) }\end{array}$ \\
\hline Total ventricle volume (mL) & 123 & 157 & $<.001^{\mathrm{d}}$ & .060 & 135 & $<.001^{\mathrm{d}}$ & 75.4 \\
\hline Total ventricle volume ratio (\%) & 8.1 & 10.5 & $<.001 \mathrm{~d}$ & $.017^{d}$ & 8.8 & $<.001^{\mathrm{d}}$ & 5 \\
\hline Bilateral ventricle volume (mL) & 114 & 147 & $<.001^{\mathrm{d}}$ & .060 & 125 & $<.001^{\mathrm{d}}$ & 66.9 \\
\hline Bilateral ventricle volume ratio (\%) & 7.5 & 9.8 & $<.001^{\mathrm{d}}$ & $.013^{\mathrm{d}}$ & 8.2 & $<.001^{\mathrm{d}}$ & 4.4 \\
\hline Total subarachnoid space volume $(\mathrm{mL})$ & 270 & 251 & .678 & $.010^{\mathrm{d}}$ & 294 & .127 & 264 \\
\hline Total subarachnoid space volume ratio (\%) & 18.0 & 16.8 & .587 & $.030^{\mathrm{d}}$ & 19.3 & .223 & 17.8 \\
\hline CSF volume of frontal convexity $(\mathrm{mL})$ & 51.1 & 41.0 & $.003^{\mathrm{d}}$ & $.016^{\mathrm{d}}$ & 52.1 & .218 & 51.1 \\
\hline $\mathrm{CSF}$ volume of parietal convexity $(\mathrm{mL})$ & 42.4 & 28.4 & $<.001^{\mathrm{d}}$ & $<.001^{\mathrm{d}}$ & 44.1 & .162 & 42.4 \\
\hline CSF volume of Sylvian fissure and basal cistern $(\mathrm{mL})$ & 119 & 121 & $.030^{\mathrm{d}}$ & .077 & 136 & $<.001^{d}$ & 119 \\
\hline CSF volume of posterior fossa $(\mathrm{mL})$ & 57.1 & 59.1 & .232 & 1.000 & 58.9 & .226 & 57.1 \\
\hline Upper-to-lower subarachnoid space ratio & 0.55 & 0.37 & $<.001^{\mathrm{d}}$ & $.008^{\mathrm{d}}$ & 0.49 & $<.001^{\mathrm{d}}$ & 0.79 \\
\hline Evans Index & 0.31 & 0.34 & $<.001^{\mathrm{d}}$ & $.028^{\mathrm{d}}$ & 0.32 & $.004^{d}$ & 0.29 \\
\hline Y-Evans Index & 0.22 & 0.23 & $.001^{\mathrm{d}}$ & .053 & 0.22 & .095 & 0.21 \\
\hline Z-Evans Index & 0.38 & 0.43 & $<.001^{d}$ & $.002^{d}$ & 0.39 & $<.001^{\mathrm{d}}$ & 0.31 \\
\hline X-Max Index & 0.63 & 0.65 & $<.001^{d}$ & .073 & 0.64 & $<.001^{d}$ & 0.60 \\
\hline Y-Max Index & 0.63 & 0.67 & $<.001^{d}$ & $.048^{d}$ & 0.64 & $.001^{\mathrm{d}}$ & 0.58 \\
\hline Z-Max Index & 0.70 & 0.74 & $<.001^{d}$ & .613 & 0.74 & $<.001^{\mathrm{d}}$ & 0.62 \\
\hline Callosal angle (degree) & 80.9 & 66.2 & $<.001^{\mathrm{d}}$ & .117 & 73.4 & $<.001^{\mathrm{d}}$ & 104 \\
\hline
\end{tabular}

Note:-Max indicates maximum.

a $P$ value?: probability value for the Mann-Whitney-Wilcoxon test between the tap-positive group and controls.

${ }^{b} P$ value ${ }^{2}$ : probability value for the Mann-Whitney-Wilcoxon test between the tap-positive and tap-negative groups.

${ }^{c} P$ value ${ }^{3}$ : probability value for the Mann-Whitney-Wilcoxon test between the tap-negative group and controls.

d Significant.

Table 3: At the maximum AUC, thresholds, sensitivity, specificity, and age-adjusted OR $(95 \% \mathrm{Cl})$ for the tap-positive compared with the tap-negative group

\begin{tabular}{|c|c|c|c|c|c|c|}
\hline Tap-Positive vs Tap-Negative & AUC & $\begin{array}{l}\text { Optimal } \\
\text { Threshold }\end{array}$ & Sensitivity & Specificity & OR $(95 \% \mathrm{Cl})$ & $P$ Value \\
\hline Total ventricle volume $(\mathrm{mL})$ & 0.657 & 155 & 58.3 & 80.0 & $1.49(1.13-1.96)$ & .004 \\
\hline Total ventricle volume ratio (\%) & 0.700 & 8.6 & 79.2 & 56.0 & $1.49(1.13-1.96)$ & .005 \\
\hline Bilateral ventricle volume (mL) & 0.657 & 147 & 54.2 & 84 & $1.52(1.15-2.07)$ & .003 \\
\hline Bilateral ventricle volume ratio (\%) & 0.705 & 7.9 & 83.3 & 56.0 & $1.40(1.06-1.84)$ & .019 \\
\hline Total subarachnoid space volume $(\mathrm{mL})^{\mathrm{b}}$ & 0.714 & 312 & 87.5 & 48.0 & $0.66(0.49-0.89)$ & .006 \\
\hline Total subarachnoid space volume ratio (\%) & 0.682 & 17.9 & 75.0 & 72.0 & $0.63(0.48-0.82)$ & $<.001$ \\
\hline CSF volume of frontal convexity $(\mathrm{mL})^{\mathrm{b}}$ & 0.701 & 45.9 & 79.2 & 76.0 & $0.58(0.45-0.74)$ & $<.001$ \\
\hline $\mathrm{CSF}$ volume of parietal convexity $(\mathrm{mL})^{\mathrm{b}}$ & 0.768 & 37.9 & 91.7 & 52.0 & $0.60(0.45-0.79)$ & $<.001$ \\
\hline CSF volume of Sylvian fissure and basal cistern $(\mathrm{mL})$ & 0.648 & 137 & 75.0 & 56.0 & $0.71(0.52-0.96)$ & .028 \\
\hline CSF volume of posterior fossa $(\mathrm{mL})$ & 0.500 & 78.7 & 16.7 & 96.0 & $1.41(0.89-2.25)$ & .146 \\
\hline Upper-to-lower subarachnoid space ratio ${ }^{b}$ & 0.723 & 0.33 & 45.8 & 92.0 & $0.62(0.46-0.83)$ & .002 \\
\hline Evans Index & 0.683 & 0.33 & 58.3 & 80.0 & $1.37(1.04-1.80)$ & .026 \\
\hline Y-Evans Index & 0.662 & 0.24 & 41.7 & 92.0 & $1.42(0.99-2.03)$ & .057 \\
\hline Z-Evans Index ${ }^{b}$ & 0.758 & 0.42 & 66.7 & 88.0 & $1.74(1.35-2.24)$ & $<.001$ \\
\hline X-Max Index & 0.640 & 0.62 & 70.8 & 60.0 & $1.55(1.12-2.16)$ & .009 \\
\hline Y-Max Index & 0.665 & 0.69 & 45.8 & 92.0 & $1.69(1.26-2.27)$ & $<.001$ \\
\hline Z-Max Index & 0.543 & 0.74 & 58.3 & 60.0 & $1.13(0.85-1.52)$ & .401 \\
\hline Callosal angle (degree) & 0.632 & 77.3 & 87.5 & 48.0 & $1.51(1.13-2.02)$ & .005 \\
\hline
\end{tabular}

a Probability value for the age-adjusted ORs in a logistic regression model

${ }^{b}$ Rows in which the AUC $>0.7$ and $P<.05$ are at the optimal threshold.

tio were significantly different between the tap-positive and tapnegative groups. The bilateral ventricular volume ratio seemed to be robust and representative of the ventriculomegaly. The ranges of the 3-directional expanding ratios for the bilateral ventricles were $0.55-0.70$ at the $\mathrm{x}$-Maximum Index, $0.49-0.71$ at the $y$ Maximum Index, and 0.36-0.81 at the z-Maximum Index; and those for the frontal horns were $0.22-0.39$ at the Evans Index, $0.17-0.26$ at the $y$-Evans Index, and $0.17-0.48$ at the $z$-Evans Index. The bilateral ventricles and their frontal horns were most expanded toward to the $\mathrm{z}$-axial direction in the tap-positive group with iNPH. The Evans Index, z-Evans Index, and y-Maximum Index were statistically significant between the tap-positive and tap-negative groups. For discriminating the tap-test response, the CSF volume of the parietal convexity was the highest index (AUC,
0.768; sensitivity, 91.7\%; specificity, 52.0\%), the z-Evans Index was the second (AUC, 0.758; sensitivity, 66.7\%; specificity, $88.0 \%$ ), and the upper-to-lower subarachnoid space ratio index was the third (AUC, 0.723 ; sensitivity, $45.8 \%$; specificity, 92.0\%), as shown in Table 3 and Fig 5 . These 3 indices remained statistically significant for discriminating the shunt-effective iNPH group from the tap-negative group (On-line Table). There was a significant relationship between the upper-to-lower subarachnoid space ratio and CSF volume of the parietal convexity $(r$, 0.680 ; $95 \%$ CI, -0.533 to $-0.788 ; P<.001)$. Furthermore, the z-Evans Index was significantly associated with the upper-tolower subarachnoid space ratio $(r,-0.527 ; 95 \% \mathrm{CI},-0.676$ to $-0.336 ; P<.001)$ or the CSF volume of the parietal convexity $(r$, $-0.238 ; 95 \% \mathrm{CI},-0.445$ to $-0.007 ; P=.004)$. If the patients with 

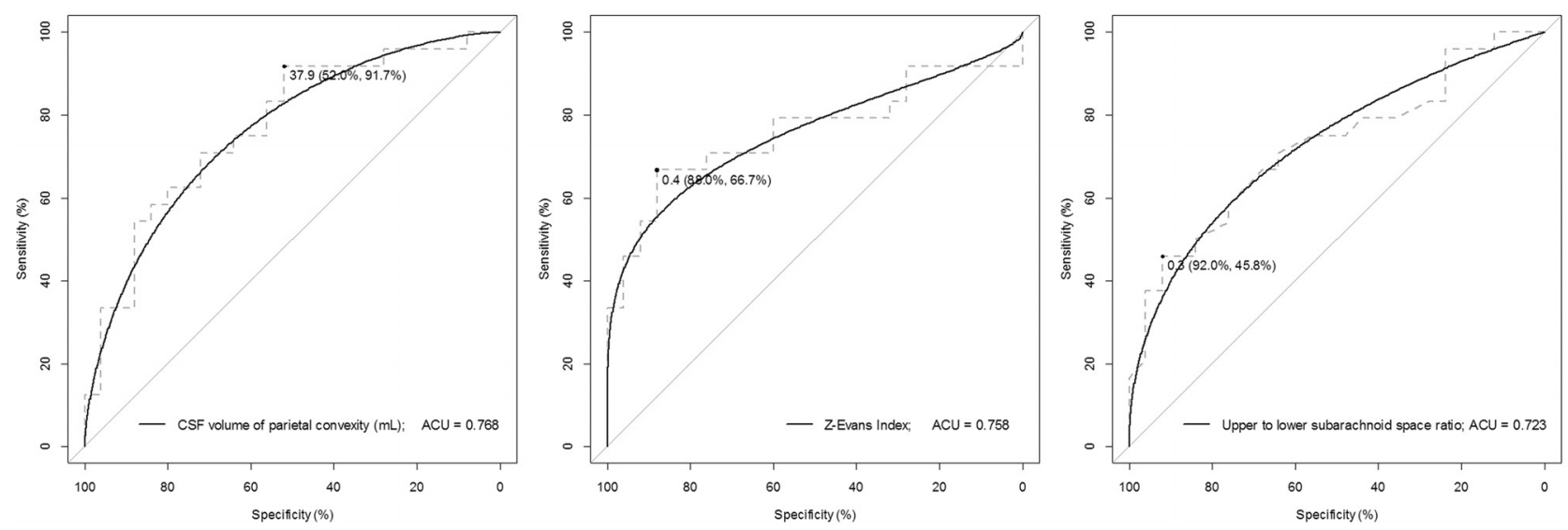

FIG 5. Receiver operating characteristic curves for discriminating tap-positive from the tap-negative group. The ROC graphs show specificity on the $x$-axis and sensitivity on the $y$-axis. The left graph shows the ROC curve of the parietal convexity of the subarachnoid space, the middle one shows that of the z-Evans Index, and the right one shows that of the upper-to-lower subarachnoid space ratio. The black circle points indicate the optimal cutoff points of the maximum area under the ROC curve.

suspected iNPH had any of the MR imaging findings - that is, the CSF volume of the parietal convexity of $<38 \mathrm{~mL}$, upper-to-lower subarachnoid space ratio of $<0.33$, and/or z-Evans Index of $>0.42$ - they had the possibility of a $>1.5$-times higher effectiveness of the CSF tap test. No useful combination of parameters obviously increased the AUC compared with the single parameter.

\section{DISCUSSION}

Our quantitative volumetric analyses determined that volume expansion of the bilateral ventricles, especially at the frontal horns, was toward the $\mathrm{z}$-axial direction, rather than the $\mathrm{x}$-axial direction, in the patients with iNPH. Therefore, we newly proposed the zEvans Index, which was a representative index for z-axial directional expansion of the frontal horns of the bilateral ventricles. We found that the z-Evans Index had the most significant relationship with the patients with iNPH responded to the tap test among the parameters of the ventricles. Because DESH or high-convexity tightness has been recognized as a highly sensitive radiologic finding for iNPH diagnosis, ${ }^{6,19}$ a coronal MR imaging section has been recommended for this diagnosis. ${ }^{6,20}$ A coronal MR imaging section is also suitable for the measurement of the z-Evans Index, the same as the DESH and callosal angle. As quantitative indices representing high-convexity tightness and the enlarged Sylvian fissure, the upper-to-lower subarachnoid space ratio and the CSF volume of the 4 segmented parts of the subarachnoid spaces were newly proposed in this study. These 2 parameters were confirmed to have a significant correlation with each other, and there was a significantly inverse correlation between the upper-to-lower subarachnoid space ratio and the $z$-Evans Index. These findings support the view that high-convexity tightness in iNPH is caused by outside compression from the $\mathrm{z}$-directional expansion of the bilateral ventricles.

Our study had some limitations. First, the highest AUC for discriminating the tap-test response was 0.768 , which was relatively low. Although differentiating the tap-positive iNPH group from the controls was simple by using the $\mathrm{z}$-Evans Index (AUC of 0.91), the same as the Evans Index (AUC of 0.84), or the callosal angle (AUC of 0.93), the Evans Index and callosal angle were not sufficiently able to estimate the tap-test response in this study. These reasons would mainly complicate iNPH diagnosis only by radiologic findings, and we emphasize that the CSF tap test is important for the diagnosis of iNPH, though the CSF tap test has high specificity $(73-100 \%)$, its sensitivity is low $(26-61 \%)$ for diagnosing shunt-responsive iNPH., ${ }^{7,21}$ The other limitation was that the signal-intensity-based CSF segmentation method based on the T2-weighted 3D-SPACE sequence had not yet been used in the field of neuroradiology, compared with the atlas-based automatic segmentation method. However, this simple segmentation method by using high-contrast CSF on a T2-weighted sequence will spread throughout the field of neuroradiology with the progression of high-field MR imaging scanners.

\section{CONCLUSIONS}

This study provides novel morphologic evidence that volume expansion of the bilateral ventricle is toward the z-axial direction, rather than the $\mathrm{x}$-axial direction, in patients with iNPH responded to the tap test. In particular, the $\mathrm{z}$-directional expansion of the frontal horn of the bilateral ventricles, named the z-Evans Index, was found to be a useful index for predicting the response to the CSF tap test. Cases of z-directional ventriculomegaly concurrent with decreased CSF volume at the parietal convexity subarachnoid space or a decreased upper-to-lower subarachnoid space ratio are thought to constitute a pivotal morphologic finding for iNPH diagnosis. These novel morphologic findings may contribute to future studies concerning the pathogenesis of iNPH underlying simultaneous enlargement of the ventricles, basal cistern, and Sylvian fissure and narrowing of the sulci at high convexity.

\section{ACKNOWLEDGMENTS}

We would like to thank Dr Yasutaka Fushimi, Department of Diagnostic Imaging and Nuclear Medicine, Kyoto University Graduate School of Medicine, for his advice concerning the MR imaging techniques; and also Professor Kazunari Ishii, Department of Radiology and Nuclear Medicine, Hyogo Brain and Heart Center, for the transfer of his original AVSIS 2013 software package by using the SPM8 software program. We would also like to thank the radiology staff of the Rakuwakai Otowa Hospital, par- 
ticularly Masatoshi Katayama, Masaru Yamazaki, and Rikiya Kikumoto.

Disclosures: Masatsune Ishikawa—RELATED: Grant: Health and Labor Sciences Research Grant on Measures for Intractable Disease: studies on the epidemiology, pathophysiology, and treatment of normal pressure hydrocephalus; UNRELATED: Payment for Lectures (including service on Speakers Bureaus): lecture fees from Medtronic Japan (plus honoraria) and Codman Japan.

\section{REFERENCES}

1. Ishikawa M; Guideline Committee for Idiopathic Normal Pressure Hydrocephalus, Japanese Society of Normal Pressure Hydrocephalus. Clinical guidelines for idiopathic normal pressure hydrocephalus. Neurol Med Chir (Tokyo) 2004;44:222-23 CrossRef Medline

2. Ishikawa M, Hashimoto M, Kuwana N, et al. Guidelines for management of idiopathic normal pressure hydrocephalus. Neurol Med Chir (Tokyo) 2008;48(suppl):S1-23 CrossRef Medline

3. Marmarou A, Bergsneider M, Relkin N, et al. Development of guidelines for idiopathic normal-pressure hydrocephalus: introduction. Neurosurgery 2005;57:S1-3; discussion ii-v Medline

4. Mori E, Ishikawa M, Kato T, et al; Japanese Society of Normal Pressure Hydrocephalus. Guidelines for management of idiopathic normal pressure hydrocephalus: second edition. Neurol Med Chir (Tokyo) 2012;52:775-809 CrossRef Medline

5. Relkin N, Marmarou A, Klinge P, et al. Diagnosing idiopathic normal-pressure hydrocephalus. Neurosurgery 2005;57(3 suppl):S4-16; discussion ii-v CrossRef Medline

6. Hashimoto M, Ishikawa M, Mori E, et al; Study of INPH on neurological improvement (SINPHONI). Diagnosis of idiopathic normal pressure hydrocephalus is supported by MRI-based scheme: a prospective cohort study. Cerebrospinal Fluid Res 2010;7:18 CrossRef Medline

7. Ishikawa M, Hashimoto M, Mori E, et al. The value of the cerebrospinal fluid tap test for predicting shunt effectiveness in idiopathic normal pressure hydrocephalus. Fluids Barriers CNS 2012;9:1 CrossRef Medline

8. Evans WA. An encephalographic ratio for estimating ventricular enlargement and cerebral atrophy. Arch NeurPsych 1942;47:931-37 CrossRef

9. Ambarki $\mathrm{K}$, Israelsson $\mathrm{H}$, Wahlin $\mathrm{A}$, et al. Brain ventricular size in healthy elderly: comparison between Evans index and volume measurement. Neurosurgery 2010;67:94-99; discussion 99 CrossRef Medline

10. Toma AK, Holl E, Kitchen ND, et al. Evans' index revisited: the need for an alternative in normal pressure hydrocephalus. Neurosurgery 2011;68:939-44 CrossRef Medline

11. Algin O, Turkbey B. Evaluation of aqueductal stenosis by 3D sampling perfection with application-optimized contrasts using different flip angle evolutions sequence: preliminary results with 3T MR imaging. AJNR Am J Neuroradiol 2012;33:740 - 46 CrossRef Medline

12. Kartal MG, Algin O. Evaluation of hydrocephalus and other cerebrospinal fluid disorders with MRI: an update. Insights Imaging 2014;5:531-41 CrossRef Medline

13. Lichy MP, Wietek BM, Mugler JP 3rd, et al. Magnetic resonance imaging of the body trunk using a single-slab, 3-dimensional, T2weighted turbo-spin-echo sequence with high sampling efficiency (SPACE) for high spatial resolution imaging: initial clinical experiences. Invest Radiol 2005;40:754-60 CrossRef Medline

14. Mugler JP 3rd. Optimized three-dimensional fast-spin-echo MRI.J Magn Reson Imaging 2014;39:745-67 CrossRef Medline

15. Gao KC, Nair G, Cortese IC, et al. Sub-millimeter imaging of brainfree water for rapid volume assessment in atrophic brains. Neuroimage 2014;100:370-78 CrossRef Medline

16. Falcão AX, Udupa JK. A 3D generalization of user-steered live-wire segmentation. Med Image Anal 2000;4:389-402 CrossRef Medline

17. Zhang $\mathrm{L}, \mathrm{Wu} \mathrm{X}$. An edge-guided image interpolation algorithm via directional filtering and data fusion. IEEE Trans Image Process 2006; 15:2226-38 CrossRef Medline

18. Fedorov A, Beichel R, Kalpathy-Cramer J, et al. 3D Slicer as an image computing platform for the quantitative imaging network. Magn Reson Imaging 2012;30:1323-41 CrossRef Medline

19. Sasaki M, Honda S, Yuasa T, et al. Narrow CSF space at high convexity and high midline areas in idiopathic normal pressure hydrocephalus detected by axial and coronal MRI. Neuroradiology 2008; 50:117-22 CrossRef Medline

20. Virhammar J, Laurell K, Cesarini KG, et al. Preoperative prognostic value of MRI findings in 108 patients with idiopathic normal pressure hydrocephalus. AJNR Am J Neuroradiol 2014;35:2311-18 CrossRef Medline

21. Kubo Y, Kazui H, Yoshida T, et al. Validation of grading scale for evaluating symptoms of idiopathic normal-pressure hydrocephalus. Dement Geriatr Cogn Disord 2008;25:37-45 CrossRef Medline

22. Wikkelsø C, Hellstrom P, Klinge PM, et al; European iNPH Multicentre Study Group. The European iNPH Multicentre Study on the predictive values of resistance to CSF outflow and the CSF tap test in patients with idiopathic normal pressure hydrocephalus. J Neurol Neurosurg Psychiatry 2013;84:562-68 CrossRef Medline 